\title{
ZERO AUTOCORRELATION WAVEFORMS: A DOPPLER STATISTIC AND MULTIFUNCTION PROBLEMS
}

\author{
John J. Benedetto, Jeffrey Donatelli, Ioannis Konstantinidis, and Christopher Shaw
}

\author{
University of Maryland \\ Department of Mathematics \\ Norbert Wiener Center \\ College Park MD 20742
}

\begin{abstract}
Constant amplitude zero autocorrelation (off the dc component) waveforms are constructed. These are called CAZAC waveforms. In the $d$-dimensional case they consist of $N$ vectors, where $N$ is given, and $N$ is generally greater than $d$. The constructions are algebraic and have been implemented in user friendly software. They have the added feature that they are a spanning set for all $d$-dimensional signals. As such, and for $N$ large, they are numerically stable in the presence of machine imperfections and they give good signal reconstruction in the presence of various noises. The one dimensional case provides effective thresholding to compute doppler shifts.
\end{abstract}

\section{INTRODUCTION}

We shall analyze a certain class of unimodular low correlation waveforms, and indicate the role they play in a multifunction environment associated with the theory of frames. In fact, the specific waveforms with which we deal are of finite length and have 0-autocorrelation off of the dc- component. Such waveforms are referred to as CAZAC waveforms, viz., Constant Amplitude Zero Autocorrelation. There is an extensive literature on CAZACs because of the importance of such waveforms in communications and coding theory, e.g., [1, 2, 3, 4, 5, 6]. Our own interest is based on the importance of waveform design in several aspects of modern radar [7, 8, 9] .

Generally, there are distinct CAZAC waveforms of any given length $K$, and different constructions of CAZACs may yield different applicability. We begin in Section 2 by defining the autocorrelation and crosscorrelation of signals of finite length $K$, as well as the ambiguity function of a given waveform of finite length. It is noted that the usual notion of the ambiguity function on the real line $\mathbb{R}$ is the analogue of our discrete definition. Then, properties of CAZACs are recorded including the important fact that $u$ is CAZAC if and only if its discrete Fourier transform $U$ is CAZAC. In particular, CAZAC waveforms are broadband, and the best finite energy approximants by waveforms of smaller bandwidth are products of $U$ by characteristic functions. The rationale for CAZACs is stated, including its role in effective matched filtering. The CAZACs we have constructed, and whose software [10] we have made available, have the property that $K$ is not square-free, and we give a typical example.

Section 3 deals with a pristine form of the Doppler effect, and we construct a statistic in order to compute frequency shifting and, therefore, target speed. This statistic is not only elementary to explain and useful and accurate, it is also based on some intriguing arithmetic complexity. We state a fundamental theoretical result as well as examples.

We give some basic results from the theory of frames in Section 4 as a prelude to our multifunction vector-valued frame waveform problem. This problem is inspired by the fact that CAZAC waveforms can be generalized as vector-valued functions defined on $\{0,1, \ldots, K-1\}$ and taking values in the unit sphere $S^{d-1}$ of $d$-dimensional Euclidean space $\mathbb{R}^{d}$. For applications in which numerical stability and resilience to machine error are important, we take $K>d$. We were also motivated to pose the multifunction vector-valued frame waveform problem because of our formulation of the notion of generalized matched filtering.

\section{CAZAC WAVEFORMS}

Let $e_{m}(n)=e^{\frac{-2 \pi i m n}{K}}$ and $\mathbb{Z}_{K}=\{0,1, \ldots, K-1\}$. Let $u, v$ : $\mathbb{Z}_{K} \rightarrow \mathbb{C}$ be $K$-periodic waveforms. The crosscorrelation of $u$ and $v$ is

$$
C_{u, v}(m)=\frac{1}{K} \sum_{k=0}^{K-1} u(m+k) \bar{\nu}(k)
$$

for $m=0,1, \ldots, K-1$.

The autocorrelation of $u$ is its crosscorelation with itself: $A_{u}(m)=C_{u, u}(m)$.

The ambiguity function of $u, A: \mathbb{Z}_{K} \times \mathbb{Z}_{K} \rightarrow \mathbb{C}$, is defined as

$$
A_{u}(j, k)=C_{u, u e_{k}}(j)=\frac{1}{K} \sum_{m=0}^{K-1} u(m+j) \overline{u(m)} e^{\frac{2 \pi i m k}{K}} .
$$


It is natural to refer to $A$ as the ambiguity function of $u$, since in the usual setting on the real line $\mathbb{R}$, the analogue ambiguity function is

$$
\begin{aligned}
A_{u}(t, \gamma) & =\int_{\widehat{\mathbb{R}}} U\left(\omega-\frac{\gamma}{2}\right) \overline{U\left(\omega+\frac{\gamma}{2}\right)} e^{2 \pi i t\left(\omega+\frac{\gamma}{2}\right)} d \omega \\
& =\int_{\mathbb{R}} u(s+t) \overline{u(s)} e^{2 \pi i s \gamma} d s,
\end{aligned}
$$

where $U$ is the Fourier transform of $u: \mathbb{R} \rightarrow \mathbb{C}, \widehat{\mathbb{R}}$ is $\mathbb{R}$ considered as the spectral domain, and the $L^{2}$ norm (finite energy) of $u$ is designated by $\|u\|_{2}$.

A $K$-periodic waveform $u$ is a constant amplitude zero autocorrelation waveform (CAZAC) if $|u(m)|=1, m=$ $0,1, \ldots, K-1$, and its autocorrelation $A_{u}(m)$ is 0 for $m=$ $1, \ldots, K-1$ (see Fig. 11). There are different constructions of different CAZAC waveforms resulting in different behavior vis à vis Doppler, additive noises, and approximation by bandlimited waveforms.

\section{Properties of CAZAC waveforms}

- $u \mathrm{CAZAC} \Rightarrow u$ is broadband (full bandwidth).

- $u$ CA $\Longleftrightarrow$ DFT of $u$ is ZAC off dc. ( DFT of $u$ can have zeros)

- $u$ CAZAC $\Longleftrightarrow$ DFT of $u$ is CAZAC.

\section{Rationale for CAZAC waveforms}

$C A$ allows transmission at peak power. In particular, the system does not have to deal with the suprise of greater than expected amplitude. Further, distortion amplitude variations can be detected using $C A$. In fact, with $C A$, amplitude variations during transmission due to additive noise can be theoretically eliminated at the receiver without distorting the message.

Example of CAZAC Waveform $K=75$ :

$u(x)=\left(1,1,1,1,1,1, e^{2 \pi i \frac{1}{15}}, e^{2 \pi i \frac{2}{15}}, e^{2 \pi i \frac{1}{5}}, e^{2 \pi i \frac{4}{15}}, e^{2 \pi i \frac{1}{3}}\right.$, $e^{2 \pi i \frac{7}{15}}, e^{2 \pi i \frac{3}{5}}, e^{2 \pi i \frac{11}{15}}, e^{2 \pi i \frac{13}{15}}, 1, e^{2 \pi i \frac{1}{5}}, e^{2 \pi i \frac{2}{5}}, e^{2 \pi i \frac{3}{5}}, e^{2 \pi i \frac{4}{5}}$, $1, e^{2 \pi i \frac{4}{15}}, e^{2 \pi i \frac{8}{15}}, e^{2 \pi i \frac{4}{5}}, e^{2 \pi i \frac{16}{15}}, e^{2 \pi i \frac{1}{3}}, e^{2 \pi i \frac{2}{3}}, e^{2 \pi i}, e^{2 \pi i \frac{4}{3}}$, $e^{2 \pi i \frac{5}{3}}, 1, e^{2 \pi i \frac{2}{5}}, e^{2 \pi i \frac{4}{5}}, e^{2 \pi i \frac{6}{5}}, e^{2 \pi i \frac{8}{5}}, 1, e^{2 \pi i \frac{7}{15}}, e^{2 \pi i \frac{14}{15}}$, $e^{2 \pi i \frac{7}{5}}, e^{2 \pi i \frac{28}{15}}, e^{2 \pi i \frac{1}{3}}, e^{2 \pi i \frac{13}{15}}, e^{2 \pi i \frac{7}{5}}, e^{2 \pi i \frac{29}{15}}, e^{2 \pi i \frac{37}{15}}, 1$, $e^{2 \pi i \frac{3}{5}}, e^{2 \pi i \frac{6}{5}}, e^{2 \pi i \frac{9}{5}}, e^{2 \pi i \frac{12}{5}}, 1, e^{2 \pi i \frac{2}{3}}, e^{2 \pi i \frac{4}{3}}, e^{2 \pi i \cdot 2}, e^{2 \pi i \frac{8}{3}}$, $e^{2 \pi i \frac{1}{3}}, e^{2 \pi i \frac{16}{15}}, e^{2 \pi i \frac{9}{5}}, e^{2 \pi i \frac{38}{15}}, e^{2 \pi i \frac{49}{15}}, 1, e^{2 \pi i \frac{4}{5}}, e^{2 \pi i \frac{8}{5}}$, $e^{2 \pi i \frac{12}{5}}, e^{2 \pi i \frac{16}{5}}, 1, e^{2 \pi i \frac{13}{15}}, e^{2 \pi i \frac{26}{15}}, e^{2 \pi i \frac{13}{5}}, e^{2 \pi i \frac{52}{15}}, e^{2 \pi i \frac{1}{3}}$, $\left.e^{2 \pi i \frac{19}{15}}, e^{2 \pi i \frac{11}{5}}, e^{2 \pi i \frac{47}{15}}, e^{2 \pi i \frac{61}{15}}\right)$.

\section{A DOPPLER STATISTIC}

In classical elementary matched filtering, an electromagnetic waveform $u$ is emitted (with carrier) from a radar. The ideal reflected signal from a target is $v(t)=a \overline{u\left(t-t_{0}\right)}, a>0$ fixed; and $t_{0}$ is to be computed. The time $t_{0}$ is proportional to target distance. If, in fact, $v(t)=a \overline{u\left(t-t_{0}\right)}$ for some $t_{0}$, then

$$
C_{v, u}\left(t_{0}\right)=\sup _{t}\left|C_{v, u}(t)\right|
$$

$C_{v, u}$ is $L^{2}(\mathbb{R})$ crosscorrelation, and the maximum system response is given by the matched filter $a \overline{\hat{u}(\gamma)} e^{-2 \pi i t_{0} \gamma}$. In the digital case, CAZACs arise since travel time depends on crosscorrelation peak, and sharp peaks obviate distortion and interference in the received waveform.

There is a fundamental Doppler tolerance problem: construct a statistic to determine an unknown Doppler frequency shift. We also want to solve this for multiple frequencies.

In order to address this problem we have the following result [11].

Theorem 1 Let $K=M \times N \times N$ and let $k=0,1, \ldots, K-$ 1. The quantity $\left|C_{u, u e_{k}}(\cdot)\right|$ is $N$-periodic as a function of $k$, i.e., there are at most $N$ different graphs of $\left|C_{u, u e_{k}}(\cdot)\right|$. Also, given $k,\left|C_{u, u e_{k}}(j)\right|=0$ for all $j \neq(-k) \bmod M N$. Further, $\sum_{j=0}^{K-1}\left|C_{u, u e_{k}}(j)\right|^{2}=1$.

Remarks The Doppler statistic $\left|C_{u, u e_{k}}(j)\right|$ is excellent and provable for detecting deodorized Doppler frequency shift [11] (see Fig. 2). Also, if one graphs only $\operatorname{Re} A(j, k)=$ $\operatorname{Re} C_{u, u e_{k}}(j)$ then the statistic sometimes fails. Further, we point out that there are unresolved "arithmetic" complexities which are affected by waveform structure and length; and that our noise analysis is ongoing.

\section{FRAMES AND MULTIFUNCTION PROBLEM}

Given $H=\mathbb{R}^{d}$ or $H=\mathbb{C}^{d}, N \geq d .\left\{x_{n}\right\}_{n=1}^{N} \subseteq H$ is a finite unit norm tight frame (FUN-TF) if each $\left\|x_{n}\right\|=1$ and, for each $x \in H$,

$$
x=\frac{d}{N} \sum_{n=1}^{N}\left\langle x, x_{n}\right\rangle x_{n} .
$$

A sequence $\left\{x_{n}\right\}_{n=1}^{N} \subseteq H$ is an A-tight frame if $\left\{x_{n}\right\}_{n=1}^{N}$ spans $H$ and $A\|x\|^{2}=\sum_{n=1}^{N}\left|\left\langle x, x_{n}\right\rangle\right|^{2}$ for each $x \in H$.

\section{Recent applications of FUN-TFs}

FUN-TFs are surprisingly applicable. They have arisen in dealing with the robust transmission of data over erasure channels such as the internet [12, 13, 14], and in both multiple antenna code design for wireless communications [15] as well as multiple description coding [16, 17, 18]. There are also recent applications of FUN-TFs in quantum detection, $\Sigma-\Delta$ quantization, and Grassmanian "min-max" waveforms, e.g., [19].

\section{Rationale for frames}

Frames give redundant signal representation to compensate for machine imperfections, to ensure numerical stability, and to minimize the effects of noise.

\section{Examples of FUN-TFs}

- Orthonormal bases, the vertices of Platonic solids, and kissing numbers (from sphere packing and error correcting codes) are $F U N-T F s$. 
- (DFT FUN-TFs) $N \times d$ submatrices of the $N \times N$ DFT matrix are $F U N-T F s$ for $\mathbb{C}^{d}$. These play a major role in finite frame $\Sigma \Delta$-quantization. The vectors

$$
\begin{gathered}
x_{m}=\frac{1}{5}\left(e^{2 \pi i m \frac{1}{8}}, e^{2 \pi i m \frac{2}{8}}, e^{2 \pi i m \frac{5}{8}}, e^{2 \pi i m \frac{6}{8}}, e^{2 \pi i m \frac{7}{8}}\right), \\
m=1, \ldots, 8,
\end{gathered}
$$

form a FUN-TF for $\mathbb{R}^{5}$.

$$
N=8, d=5 \quad \frac{1}{\sqrt{5}}\left[\begin{array}{cccccccc}
* & * & \cdot & \cdot & * & * & * & . \\
* & * & \cdot & \cdot & * & * & * & \cdot \\
* & * & \cdot & \cdot & * & * & * & . \\
* & * & \cdot & \cdot & * & * & * & \cdot \\
* & * & \cdot & \cdot & * & * & * & \cdot \\
* & * & \cdot & \cdot & * & * & * & . \\
* & * & \cdot & \cdot & * & * & * & \cdot \\
* & * & \cdot & \cdot & * & * & * & \cdot
\end{array}\right]
$$

\section{The frame force}

The frame force $F: S^{d-1} \times S^{d-1} \backslash D \rightarrow \mathbb{R}^{d}$ is defined as $F(a, b)=\langle a, b\rangle(a-b)$, where $S^{d-1}$ is the unit sphere in $\mathbb{R}^{d}$. $F$ is a (central) conservative force field. The total potential energy for the frame force of $\left\{x_{n}\right\}_{n=1}^{N} \subseteq S^{d-1}$ is

$$
P=\sum_{m=1}^{N} \sum_{n=1}^{N}\left|\left\langle x_{m}, x_{n}\right\rangle\right|^{2} .
$$

Let $N \geq d$. It can be shown that the minimum value of $P$ for the frame force $F$ and $N$ variables is $\frac{N}{d}$; and the minimizers of $P$ are precisely all of the FUN-TFs of $N$ elements in $S^{d-1}[20]$.

Because of the aforementioned applications it is important to have a reliable, systematic way of computing these frames

\section{Multifunction vector-valued frame waveform problem}

Construct, code, and implement (user-friendly) $N$-periodic waveforms $(N \geq d)$

$$
\begin{aligned}
u: \mathbb{Z}_{N} & \rightarrow S^{d-1} \subseteq \mathbb{R}^{d}\left(\text { or } \mathbb{C}^{d}\right) \\
n & \rightarrow u_{n}=\left(u_{n}(1), u_{n}(2), \ldots, u_{n}(d)\right) \\
n & =0,1, \ldots, N-1
\end{aligned}
$$

which are FUN-TFs and CAZAC, i.e., each

$$
\begin{gathered}
x=\frac{d}{N} \sum_{n=0}^{N-1}\left\langle x, u_{n}\right\rangle u_{n} \text { and } A_{u}(m)=\frac{1}{N} \sum_{j=0}^{N-1}\left\langle u_{m+j}, u_{j}\right\rangle=0, \\
m=1, \ldots N-1 .
\end{gathered}
$$

\section{Acknowledgements}

This material is based upon work supported by the National Science Foundation under Grant No. DMS0504924 (first author) and VIGRE Grant No. DMS0240049 (second author), the Department of the Navy, Office of Naval Research under Grant No. N00014-02-1-0398 (first author), and the Air Force Research Laboratory, Office of Scientific Research (first and fourth authors).

\section{REFERENCES}

[1] D. C. Chu, "Polyphase codes with good periodic correlation properties," IEEE Trans. Inform. Theory, vol. 18, pp. 531-532, 1972.

[2] R. L. Frank and S. A. Zadoff, "Phase shift pulse codes with good periodic correlation properties," IEEE Trans. Inform. Theory, vol. 8, pp. 381-382, 1962.

[3] T. Helleseth and P. V. Kumar, Sequences with low correlation, chapter 21, Elsevier Publishers, 1998.

[4] A. Milewski, "Periodic sequences with optimal properties for channel estimation and fast start-up equalization," IBM J. Res. Dev., vol. 27, pp. 426-430, 1983.

[5] U. H. Rohrs and L. P. Linde, "Some unique properties and applications of perfect squares minimum phase cazac sequences," in Proc. South African Symp. on Communications and Signal Processing, 1992, pp. 155160 .

[6] R. Turyn, Sequences with small correlations, pp. 195228, John Wiley \& Sons, Inc., New York, 1968.

[7] J. R. Klauder, "The design of radar signals having both high range resolution and high velocity resolution," Bell System Technical Journal, vol. 39, pp. 809-820, 1960.

[8] J. R. Klauder, A. C. Price, S. Darlington, and W. J. Albersheim, "The theory and design of chirp radars," Bell System Technical Journal, vol. 39, pp. 745-808, 1960.

[9] N. Levanon and E. Mozeson, Radar Signals, Wiley, 2004.

[10] J. J. Benedetto and J. F. Ryan, "Software package for CAZAC code generators and Doppler shift analysis," 2004, www.math.umd.edu/ jjb/cazac.

[11] J. J. Benedetto and J. Donatelli, "A doppler statistic associated with low correlation waveforms of finite length," .

[12] P. G. Casazza and J. Kovačević, "Equal-norm tight frames with erasures," Adv. Comput. Math., vol. 18, no. 2-4, pp. 387-430, 2003. 


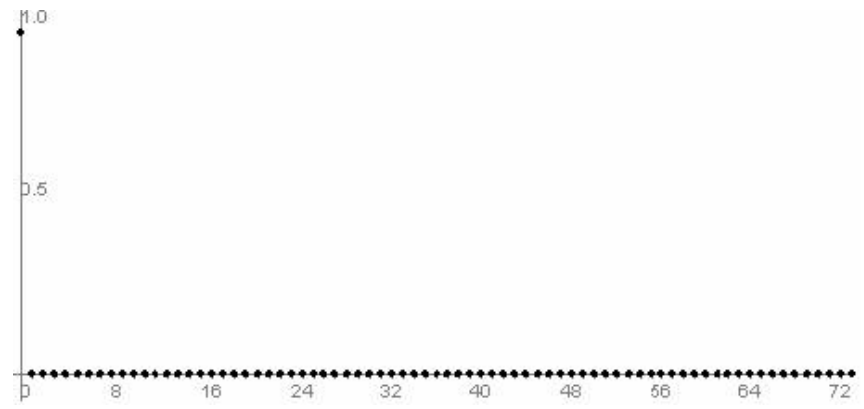

Fig. 1. Autocorrelation $A(u, u)$ for CAZAC with $K=75$.

[13] V. K. Goyal, J. Kovačević, and J. A. Kelner, "Quantized frame expansions with erasures," Appl. Comput. Harmon. Anal., vol. 10, no. 3, pp. 203-233, 2001.

[14] R. B. Holmes and V. I. Paulsen, "Optimal frames for erasures," Linear Algebra Appl., vol. 377, pp. 31-51, 2004.

[15] B. M. Hochwald, T. L. Marzetta, T. J. Richarson, W. Sweldens, and R. L. Urbanke, "Systematic design of unitary speace-time constellations," IEEE Trans. Inform. Theory, vol. 46, no. 6, pp. 1962-1973, 2000.

[16] T. Strohmer and R. W. Jr. Heath, "Grassmannian frames with applications to coding and communication," Appl. Comp. Harmon. Anal., vol. 14, no. 3, pp. 257-275, 2003.

[17] V. K. Goyal, J. Kovačević, and M. Vetterli, "Multiple description transform coding: Robustness to erasures using tight frame expansions," Proc. IEEE Int. Symp. on Inform. Theory, p. 408, 1998.

[18] V. K. Goyal, J. Kovačević, and M. Vetterli, "Quantized frame expansions as source-channel codes for erasure channels," Proc. IEEE Data Compression Conf., pp. 326-335, 1999.

[19] J. J. Benedetto, A. Powell, and Ö. Yilmaz, " $\Sigma-\Delta$ quantization and finite frames," IEEE Trans. Inform. Theory, 2004, Submitted.

[20] J. J. Benedetto and M. Fickus, "Finite normalized tight frames," Adv. Comp. Math., vol. 18, no. 2-4, pp. 357$385,2003$.

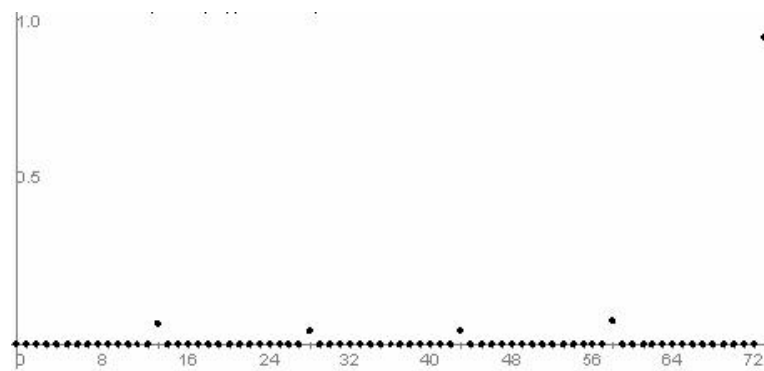

(a)

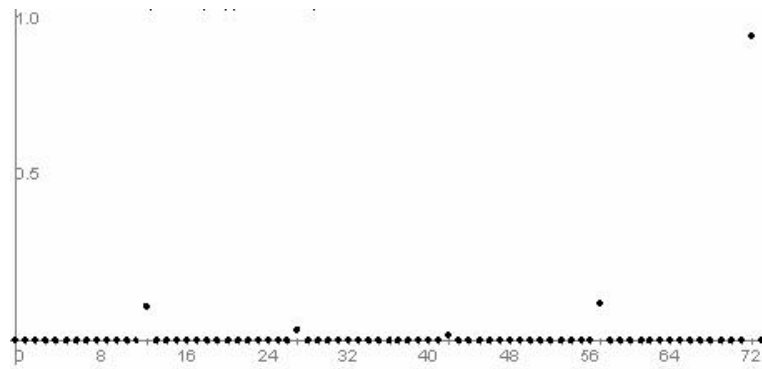

(b)

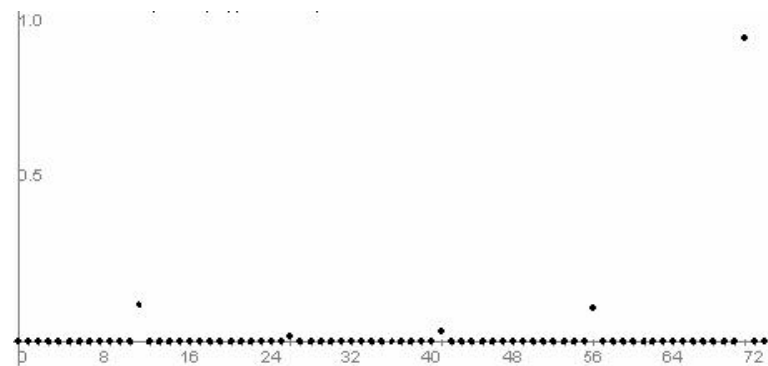

(c)

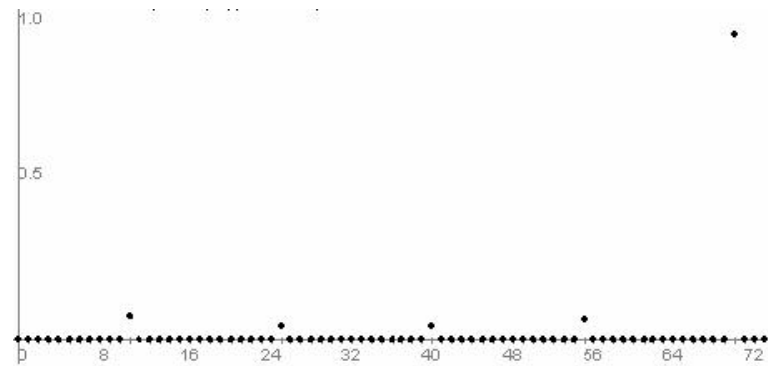

(d)

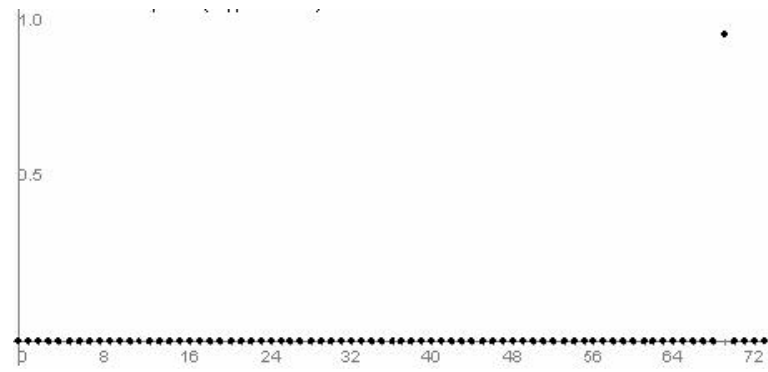

(e)

Fig. 2. Doppler statistic for CAZAC with $K=75$; (a) - (e) graphs of $\left|C_{u, u e_{k}}(\cdot)\right|$ for $k=1, \ldots, 5$, respectively. 